\title{
Large $N$ twisted partition functions in 3d-3d correspondence and holography
}

\author{
Dongmin Gang \\ Center for Theoretical Physics, Seoul National University, Seoul 08826, Korea
}

Nakwoo Kim

Department of Physics and Research Institute of Basic Science, Kyung Hee University, Seoul 02447, Korea and School of Physics, Korea Institute for Advanced Study, Seoul 02445, Korea

(Received 22 August 2018; published 23 January 2019)

\begin{abstract}
We study the large $N$ limit of twisted partition functions on $\mathcal{M}_{g, p}$, the $S^{1}$ bundle of degree $p$ over a Riemann surface of genus $g$, for $3 \mathrm{D} \mathcal{N}=2$ superconformal field theories arising as low-energy limit of wrapped $N$ M5-branes on hyperbolic 3-manifold $M$. We study contributions from two Bethe vacua which correspond to two canonical irreducible $S L(N, \mathbb{C})$ flat connections on $M$ via 3D-3D correspondence. Using mathematical results on perturbative Chern-Simons invariants around the flat connections, we find universal expressions for the large $N$ twisted partition functions contributed from the two Bethe vacua in term of the hyperbolic volume of $M$. The two large $N$ partition functions perfectly match the on-shell actions for two Bolt-type solutions in the holographic dual $\mathrm{AdS}_{4}$ gravity, respectively.
\end{abstract}

DOI: 10.1103/PhysRevD.99.021901

\section{INTRODUCTION AND SUMMARY}

As a consistent theory of quantum gravity, string/Mtheory is expected to provide microscopic understandings of quantum aspects of black holes (BHs). In a celebrated work [1], this hope was realized for extremal black holes in Minkowski spacetime, by reproducing the BekensteinHawking entropy through counting D-brane bound states. Recently, this success was extended to black holes in asymptotically anti-de-Sitter spacetime. In particular, the entropy of magnetically charged supersymmetric black holes in $\mathrm{AdS}_{4}$ supergravity can be explained using the holographic principle [2-11]. The AdS/CFT correspondence [12] says quantum gravity in asymptotically $\mathrm{AdS}_{4}$ spacetime should be dual to a conformal field theory (CFT) on the 3-dimensional (3D) boundary. The field theories of interest have $\mathcal{N} \geq 2$ supersymmetry, and the black hole entropy on the gravity side turns out to be related to the socalled topologically twisted indices [13-16] in the dual field theory. They are the partition functions (ptns) on supersymmetric curved backgrounds $\mathcal{M}_{g, p=0}:=\Sigma_{g} \times S^{1}$, with an appropriately chosen background magnetic flux coupled to R-symmetry current. The magnetic flux is turned on along the Riemann surface $\Sigma_{g}$ of genus $g$. The twisted indices can be then computed using the supersymmetric

Published by the American Physical Society under the terms of the Creative Commons Attribution 4.0 International license. Further distribution of this work must maintain attribution to the author(s) and the published article's title, journal citation, and DOI. Funded by SCOAP ${ }^{3}$. localization technique. Recently, the realm of localizable 3D manifolds has been further extended in [17], and we now have formulas for twisted partition functions $Z_{g, p}$, i.e., the ptn on degree- $p S^{1}$-bundles $\mathcal{M}_{g, p}$ [see (4)] over $\Sigma_{g}$.

In this paper, we study holographic duality for a large class of 3D $\mathcal{N}=2$ SCFTs $T_{N}[M]$, defined in (2) [see also (3)], arising from wrapped M5-branes on closed hyperbolic 3-manifolds $M$. The 3D theory is characterized by $N$, number of M5-branes, and the choice of a 3-manifold $M$. For each $M$, there is an associated $\mathrm{AdS}_{4} / \mathrm{CFT}_{3}$ correspondence. The holographic dual of the wrapped M5-brane 3D SCFT was studied in $[18,19]$. Since there are infinitely many such 3-manifolds [20], the wrapped M5-branes system provides a huge set of $\mathrm{AdS}_{4} / \mathrm{CFT}_{3}$ examples. We probe the holography using the twisted ptns. For $p=0$, the ptn becomes a twisted index which counts ground states of M5-branes on $\Sigma_{g} \times M$. The counting is holographically dual to the microstates counting for a supersymmetric $\mathrm{BH}$ solution interpolating the asymptotic $\mathrm{AdS}_{4}$ and its near-horizon limit $\mathrm{AdS}_{2} \times \Sigma_{g}[11]$.

The 3D-3D correspondence [21-23] provides a novel way of analyzing the $3 \mathrm{D} \mathcal{N}=2$ SCFTs. Schematically, the correspondence says

$$
\begin{aligned}
& \text { (supersymmetric ptns of } 3 \mathrm{D} T_{N}[M] \text { theory) } \\
& =(S L(N, \mathbb{C}) \text { Chern-Simons theory invariants on } M) .
\end{aligned}
$$

Refer to [24-31] for more details on 3D-3D dictionary. Via the correspondence, some supersymmetric quantities of 3D $T_{N}[M]$ theory can be evaluated without relying on a field 
TABLE I. 3d-3d dictionaries for basic ingredients in twisted partition function computation. $S_{n=0,1}^{\alpha}$ are perturbative invariants of the complex Chern-Simons theory around a flat-connection $\mathcal{A}^{\alpha}$, see Eq. (15).

\begin{tabular}{lc}
\hline \hline 3D $T_{N}[M]$ theory & $S L(N, \mathbb{C})$ CS theory \\
\hline Bethe vacuum $\alpha$ & Irreducible flat connection $\mathcal{A}^{\alpha}$ \\
Handle gluing operator $\mathcal{H}^{\alpha}$ & $\exp \left(-2 S_{1}^{\alpha}\right)$ \\
Fibering operator $\mathcal{F}^{\alpha}$ & $\exp \left(i S_{0}^{\alpha} /(2 \pi)\right)$ \\
\hline \hline
\end{tabular}

theoretic description of the 3D SCFT. For example, as summarized in Table I, the twisted partition function $Z_{g, p}$ can be written in terms of basic perturbative invariants of the complex Chern-Simons (CS) theory. These invariants are mathematically well defined and have been extensively studied in math literature.

Combining the 3D-3D dictionary with mathematical results, we obtain the large $N$ behavior of the twisted partition functions contributed from two distinguished Bethe vacua in the $T_{N}[M]$ theory. The Bethe vacua of our interest correspond to two irreducible $S L(N, \mathbb{C})$ flat connections on $M$ in 3D-3D dual complex CS theory. These two flat connections also have a natural interpretation in terms of hyperbolic geometry, see Eq. (18). They also give global minimum and maximum of the absolute value of the fibering operator $\mathcal{F}$ appearing in the ptn computation (12), see (20). We confirm that the large $N$ twisted ptns from the two Bethe-vacua nicely match the onshell action for the two Bolt-type solutions [10] in the gravity dual respectively. The comparison is summarized in Table II, which is the main point of this paper.

\section{3D $T_{N}[M]$ THEORY}

A large class of 3D $\mathcal{N}=2$ SCFTs can be engineered through a twisted compactification of 6-dimensional SCFTs. They are labelled by the internal 3-manifolds $M$ :

$T_{N}[M]:=($ Effective $3 \mathrm{D} \mathcal{N}=2$ SCFT obtained from

a twisted compactification of $6 \mathrm{D} A_{N-1}(2,0)$ theory

on a 3-manifold $M$ ).

For simplicity, we assume $M$ is a closed (compact) hyperbolic 3-manifold without boundary. To preserve supersymmetry, we perform a partial topological twisting along the internal 3-manifold using $S O(3)$ vector subgroup of $S O(5)$ R-symmetry of the $6 \mathrm{D}$ theory. The twisting preserves a quarter of supersymmetries and the resulting 3D theory becomes a 3D $\mathcal{N}=2$ SCFT with 4 supercharges. The $6 \mathrm{D}$ theory describes the low-energy effective world-volume theory of $N$ coincident M5-branes in M-theory, and the 3D theory can be considered as an effective world-volume theory of $N$ coincident M5-branes wrapped on the compact 3-manifold $M$, i.e.,

$$
\begin{aligned}
& \text { Ncoincident M5-brances on } \mathbb{R}^{3} \times M \\
& \text { in M-theory on } \mathbb{R}^{3} \times\left(T^{*} M\right) \times \mathbb{R}^{2} \\
& \text { IR world-volume theory of M5-branes } \\
& \stackrel{\longrightarrow}{\longrightarrow} T_{N}[M] \text { on } \mathbb{R}^{3} \text {. }
\end{aligned}
$$

Here $T^{*} M$ is the cotangent bundle of $M$, which is a local Calabi-Yau.

Let us comment on a subtle point in the setup. As emphasized in [32], in taking the twisted compactification we need to choose a connected subset of the vacuum moduli-space of the theory defined on $\mathbb{R}^{3}$, in order to have a genuine 3D SCFT. For a hyperbolic $M$, there is a natural choice (which is actually a single point) which is expected to become a discrete set of vacua when the theory is put on $\mathbb{R}^{2} \times S^{1}$. This discrete set of vacua corresponds to a subset of irreducible $S L(N, \mathbb{C})$ flat connections on $M$. A field theoretic construction of the effective 3D gauge theory is proposed in [32], extending the beautiful construction in $[23,33]$ for cusped 3-manifolds with at least one torus boundary component, by incorporating gauge theoretical operations corresponding to Dehn filling (removing torus boundaries) operations on 3-manifold.

\section{III. $T_{N}[M]$ ON $\mathcal{M}_{g, p}$ FOR EVEN $p$}

We now turn to the case where $T_{N}[M]$ is put on a large class of nontrivial backgrounds $\mathcal{M}_{g, p}$ [17]:

$$
\begin{aligned}
& \mathcal{M}_{g, p}:=\left(S^{1} \text {-bundle of degree } p\right. \\
& \left.\quad \text { over a Riemmann surface } \Sigma_{g} \text { of genus } g\right), \\
& \text { i.e. } S^{1} \stackrel{p}{\rightarrow} \mathcal{M}_{g, p} \rightarrow \Sigma_{g} .
\end{aligned}
$$

The metric can be written as

TABLE II. The M-theory is holographic dual to 3D $T_{N}[M]$ theory while the complex Chern-Simons theory is $3 \mathrm{D}$ 3D dual to the $T_{N}[M]$ theory. The $4 \mathrm{~d}$ Newton constant $G_{4}$ is given in (11).

\begin{tabular}{lc}
\hline \hline M-theory on $\mathrm{AdS}_{4} \times M \times \tilde{S}^{4}$ & $S L(N, \mathbb{C})$ CS theory on $M$ \\
\hline Bolt + solution $I_{g, p}^{\text {Bolt }_{+}}=\frac{\pi(4(1-g)-p)}{8 G_{4}}$ & Flat connection $\mathcal{A}_{N}^{\bar{g} \text { gem }} F_{g, p}^{\bar{g} \text { geom }}=\frac{4(1-g) N^{3}-p N^{3}}{12 \pi} \operatorname{vol}(M)$ \\
Bolt_s solution $I_{g, p}^{\text {Bolt }}=\frac{\pi(4(1-g)+p)}{8 G_{4}}$ & Flat connection $\mathcal{A}_{N}^{\text {geom }} F_{g, p}^{\text {geom }}=\frac{4(1-g) N^{3}+p N^{3}}{12 \pi} \operatorname{vol}(M)$ \\
\hline \hline
\end{tabular}




$$
d s^{2}=\beta^{2}(d \psi-p a(z, \bar{z}))^{2}+2 g_{z \bar{z}} d z d \bar{z},
$$

where $z, \bar{z}$ are local coordinates on the Riemann surface and $\psi \sim \psi+2 \pi$ parametrizes the $S^{1}$-fiber of length $\beta . a$ is a 1 -form on $\Sigma_{g}$ whose curvature $F_{a}:=d a$ is normalized as

$$
\frac{1}{2 \pi} \int_{\Sigma_{g}} d a=1
$$

To preserve some supersymmetry, we turn on the following background gauge field coupled to $U(1)$ R-symmetry.

$$
A^{R}=\beta \nu_{R}(d \psi-p a)+n_{R}\left(\pi^{*} a\right),
$$

with proper quantization conditions for $\left(\nu_{R}, n_{R}\right)$ [10]. Here $\pi^{*} a$ is a 1-form on $\mathcal{M}_{g, p}$ given as the pull-back of $a$ using the projection map $\pi: \mathcal{M}_{g, p} \rightarrow \Sigma_{g}$.

For later comparison with the bolt-type solutions in the supergravity, we follow [10] and choose

$$
\nu_{R}=\frac{1}{2}, \quad n_{R}=\frac{p}{2}+g-1, \quad p \in 2 \mathbb{Z} .
$$

Throughout the paper, we restrict our attention to the choice in (8) and some formulas below may not work for other cases. For example, the large $N$ computation in Table II give incorrect answer for the usual round $S^{3}$ case which is $\mathcal{M}_{g=0, p=1}$.

For small $N$ the effective $3 \mathrm{~d}$ theory $T_{N}[M]$ might witness emergent symmetries in addition to R-symmetry, as pointed out in [32,34]. When $N$ is large enough, on the other hand, there is no accidental symmetry and the $U(1)$ R-symmetry in the IR should be simply inherited from the compact $S O(2)$ subgroup of $S O(5)$ R-symmetry in the $6 \mathrm{D}$ theory. It implies that the $U(1)$ R-charge, $R$, should be properly quantized

$$
R(\mathcal{O}) \in \mathbb{Z}, \quad \text { for any state } \mathcal{O} \text { of } T_{N}[M] \text { on } \Sigma_{g}
$$

The Dirac quantization conditions for the $U(1)$ R-symmetry flux on $\Sigma_{g}$ are

$$
\begin{aligned}
& R(\mathcal{O}) \times n_{R}=R(\mathcal{O}) \times\left(\frac{p}{2}+g-1\right) \in \mathbb{Z}, \\
& \quad \text { for any state } \mathcal{O} \text { of } T_{N}[M] \text { on } \Sigma_{g} .
\end{aligned}
$$

From (9), we see that the Dirac quantizations are always satisfied for even $p$. In summary, for large enough $N$ we can put the $3 \mathrm{D} T_{N}[M]$ theory on any $\mathcal{M}_{g \in \mathbb{Z}_{\geq 0}, p \in 2 \mathbb{Z}}$ with supersymmetry preserving background gauge field, given in (7) and (8), coupled to the R-symmetry in the IR.

\section{HOLOGRAPHIC DUAL OF $T_{N}[M]$}

The gravity dual description is given by the uplift of a certain magnetically charged $\mathrm{AdS}_{4}$ solution in the maximally supersymmetric $D=7$ gauged supergravity $[18,19]$. Schematically, the $D=11$ solution is a product of $\mathrm{AdS}_{4}$, hyperbolic 3-manifold $M$, and a squashed 4-sphere $\tilde{S}^{4}$. Consistency of the truncation from $D=11$ down to minimal $\mathcal{N}=2, D=4$ gauged supergravity is established in [35] and it is guaranteed that we may replace the $\mathrm{AdS}_{4}$ part with any nontrivial $D=4$ solution and we still have an exact $D=11$ solution.

The computation of holographic free energy can be also first done in $D=4$ setup, and substitute the Newton constant with [36]

$$
G_{4}=\frac{3 \pi^{2}}{2 N^{3} \operatorname{vol}(M)} .
$$

Here, the hyperbolic volume is defined as

$$
\begin{aligned}
& \operatorname{vol}(M)=(\text { hyperbolic volume of } M) \\
& \quad:=(\text { volume measured in the unique hyperbolic metric }) .
\end{aligned}
$$

The hyperbolic metric is normalized as $R_{\mu \nu}=-2 g_{\mu \nu}$. Mostow's rigidity theorem [37] guarantees the uniqueness of the hyperbolic metric and thus the volume is actually a topological invariant.

As gravity duals of the boundary theory put on $\mathcal{M}_{g, p}$, we utilize the supersymmetric AdS-Taub-NUT and bolt solutions constructed in [38]. Since these solutions have nonvanishing Maxwell field, which in $D=11$ uplift appears as a twisting of the R-symmetry angle in $\tilde{S}^{4}$, one might worry about a conflict with the quantization condition $g, p$. But it turns out, since the R-symmetry angle is part of $\tilde{S}^{4}$ and we have a standard periodicity of $2 \pi$, the regularity condition for $D=4 \mathrm{NUT} /$ Bolt is enough. This is in line with the field theory side discussion, in particular (9). A comment is in order here, in comparison with the uplifts involving Sasaki-Einstein 7-manifolds. In that case, the periodicity of the R-symmetry angle from the regularity of Bolt solution should be compatible with the periodicity condition due to collapsing cycles in the Kähler-Einstein base manifold of the Sasaki-Einstein space. The readers are referred to [10] for more details, where the authors considered an explicit example of Sasaki-Einstein manifolds such as $V^{5,2}=S O(5) / S O(3)$.

\section{TWISTED PARTITION FUNCTIONS OF $T_{N}[M]$ IN 3D-3D CORRESPONDENCE}

The twisted partition function $Z_{g, p}$ on the $\mathcal{M}_{g, p}$ for general 3D $\mathcal{N}=2$ SCFTs is given as the following finite sum $[17,29]$ 


$$
Z_{g, p}=\sum_{\alpha} Z_{g, p}^{\alpha}:=\sum_{\alpha}\left(\mathcal{H}^{\alpha}\right)^{g-1}\left(\mathcal{F}^{\alpha}\right)^{p}
$$

Here $\alpha$ labels the so-called Bethe vacua [39] of the 3D theory. It is obtained by extremizing the effective $2 \mathrm{~d}$ twisted superpotential in the compactification on $\mathbb{R}^{2} \times S^{1}$. The number of vacua is equal to the Witten index $[40,41]$ of the 3D SCFT. $\mathcal{H}$ and $\mathcal{F}$ are called handle-gluing and fibering operators respectively. The explicit forms of $\mathcal{H}$ and $\mathcal{F}$ for any given ultraviolet (UV) Lagrangian are available in [17]. Let us emphasize that, the formula (12) applied to the case of $S^{3}$ partition function which corresponds to $(g, p)=$ $(0,1)$ is apparently different from the more familiar Coulmob branch integral expression [42]. But their equivalence is illustrated for a number of examples in [17].

Now let us specialize to the $T_{N}[M]$ theories in (2). The twisted ptns for these theories can be analyzed using the 3D-3D dictionaries summarized in Table I. Twisted ptns in 3D-3D correspondence were studied in $[29,30]$. In the table, the $\left\{S_{n}^{\alpha}\right\}_{n=0}^{\infty}$ represent terms in the loop expansion of the complex CS partition function around a flat-connection $\mathcal{A}^{\alpha}[43-46]$ :

$$
\begin{aligned}
Z_{\mathrm{CS} \text { pert }}^{\alpha} & :=\int \frac{D(\delta \mathcal{A})}{(\text { gauge })} e^{-\frac{1}{2 \hbar} \operatorname{CS}\left[\mathcal{A}^{\alpha}+\delta \mathcal{A} ; M\right]} \\
& \stackrel{\text { as } \hbar \text { goes to } 0}{\longrightarrow} \exp \left(\frac{1}{\hbar} S_{0}^{\alpha}+S_{1}^{\alpha}+\cdots+\hbar^{n-1} S_{n}^{\alpha}+\ldots\right) .
\end{aligned}
$$

The Chern-Simons functional is

$$
\mathrm{CS}[\mathcal{A}, M]:=\int_{M} \operatorname{Tr}\left(\mathcal{A} \wedge d \mathcal{A}+\frac{2}{3} \mathcal{A}^{3}\right) .
$$

Note that the counterpart of $\mathcal{F}$ and $\mathcal{H}$ are simply tree level and one-loop contributions in perturbation theory. More explicitly, the perturbative coefficients are given as

$$
\begin{aligned}
S_{0}^{\alpha} & =-\frac{1}{2} C S\left[\mathcal{A}^{\alpha}, M\right], \\
S_{1}^{\alpha} & :=\frac{1}{2} \log \operatorname{Tor}_{R=\text { adjoint }}\left[\mathcal{A}^{\alpha}, M\right] .
\end{aligned}
$$

$\operatorname{Tor}_{R}\left[\mathcal{A}^{\alpha}, M\right]$ is the Ray-Singer torsion of an associated vector bundle in a representation $R \in \operatorname{Hom}[S L(N, \mathbb{C}) \rightarrow$ $\left.G L\left(V_{R}\right)\right]$ twisted by a flat connection $\mathcal{A}^{\alpha}$. Here $V_{R}$ is the vector space for representation $R$ and $G L\left(V_{R}\right)$ is the general linear group on the $V_{R}$. The analytic torsion is defined as follows $[45,47,48]$

$$
\operatorname{Tor}_{R}\left[\mathcal{A}^{\alpha}, M\right]:=\frac{\left[\operatorname{det}^{\prime} \Delta_{0}\left(R, \mathcal{A}^{\alpha}\right)\right]^{3 / 2}}{\left[\operatorname{det}^{\prime} \Delta_{1}\left(R, \mathcal{A}^{\alpha}\right)\right]^{1 / 2}} .
$$

Here $\Delta_{n}\left(R, \mathcal{A}^{\alpha}\right)$ is a Laplacian acting on $V_{R}$-valued $n$-form twisted by a flat connection $\mathcal{A}^{\alpha}$. $\operatorname{det}^{\prime} \Delta$ denotes the zeta function regularized determinant of the Laplacian $\Delta$. For the one-loop part, the denominator comes from gauge field fluctuations $\delta \mathcal{A}$ while the numerator comes from the ghosts associated to a gauge fixing [49].

The 3D-3D dictionary in Table I can be derived combining several known results in literatures. The Bethe-vacua (vacua on $\mathbb{R}^{2} \times S^{1}$ ) of 3D $T_{N}[M]$ theory are in one-to-one correspondence to a subset of irreducible flatconnections on $M$ [21,32]. According to a dictionary of 3D$3 \mathrm{D}$ relation, the asymptotic expansion $Z_{\mathrm{CS} \text { pert }}^{\alpha}$ in (14) is equal to the perturbative expansion of holomorphic block $B^{\alpha}(q)[21,27]$ associated to the Bethe-vacuum $\alpha$ in the limit $q \rightarrow 1$,

$$
\begin{aligned}
& Z_{\mathrm{CS} \text { pert }}^{\alpha}(\hbar) \simeq B^{\alpha}\left(q:=e^{\hbar}\right), \\
& \quad \text { as an asymptotic expansion in } \hbar \rightarrow 0 .
\end{aligned}
$$

For general 3D $\mathcal{N}=2$ theory, the asymptotic expansion coefficients $S_{0}$ and $S_{1}$ of holomorphic block are related to the operators $\mathcal{F}$ and $\mathcal{H}$ as given in Table I [50,51].

\section{LARGE N TWISTED PARTITION FUNCTIONS AROUND TWO BETHE-VACUA AND ITS HOLOGRAPHIC DUAL}

For every hyperbolic 3-manifold $M$, there are two characteristic irreducible $S L(N, \mathbb{C})$ flat connections $\mathcal{A}_{N}^{\text {geom }}$ and $\mathcal{A}_{N}^{\overline{\text { geom }}}$ which can be constructed from the hyperbolic structure on $M$,

$\mathcal{A}_{N}^{\text {geom }}:=\rho_{N} \cdot(\omega+i e), \quad \mathcal{A}_{N}^{\overline{\text { geom }}}:=\rho_{N} \cdot(\omega-i e)$.

Here $\omega$ and $e$ are respectively the spin-connection and vielbein of the unique hyperbolic metric on $M$. They are both locally so(3)-valued 1-forms and the complex combinations $\omega \pm i e$ form an $S L(2, \mathbb{C})$ flat-connections on $M . \rho_{N}$ is the $N$-dimensional irreducible representation of $\operatorname{sl}(2, \mathbb{C})=s u(2)_{\mathbb{C}}$, and obviously $\rho_{N} \cdot(\omega \pm i e)$ become also irreducible $S L(N, \mathbb{C})$ flat connections. A crucial property of these two flat-connections is that they take the minimum (maximum) value of $\operatorname{Im}\left[S_{0}\right]$ among all $S L(N, \mathbb{C})$ flat connections. Namely,

$$
\operatorname{Im}\left[S_{0}^{\overline{\text { geom }}}\right]<\operatorname{Im}\left[S_{0}^{\alpha}\right]<\operatorname{Im}\left[S_{0}^{\text {geom }}\right],
$$

for any flat connection $\mathcal{A}^{\alpha}$ which is neither $\mathcal{A}^{\overline{\mathrm{geom}}}$ nor $\mathcal{A}^{\text {geom }}$. Combined with the 3D-3D dictionary in Table I, it is implied

$$
\left|\mathcal{F}^{\text {geom }}\right|<\left|\mathcal{F}^{\alpha}\right|<\left|\mathcal{F}^{\overline{\text { geom }}}\right|,
$$

for any Bethe-vacuum $\alpha$ which is neither $(\overline{\mathrm{geom}})$ nor (geom).

Classical actions $S_{0}^{\alpha}$ for the two connections above can be computed as follows 


$$
\begin{aligned}
\operatorname{Im}\left[S_{0}^{\text {geom }}\right]=-\frac{1}{2} \operatorname{Im}\left[C S\left(\mathcal{A}_{N}^{\text {geom }}\right)\right] & =-\frac{1}{2} \operatorname{Im}\left[C S\left(\rho_{N} \cdot(\omega+i e)\right)\right] \\
& =-\frac{1}{2} \frac{\operatorname{Tr}\left[\rho_{N} \cdot\left(T^{a}\right) \rho_{N} \cdot\left(T^{b}\right)\right]}{\operatorname{Tr}\left[T^{a} T^{b}\right]} \operatorname{Im}[C S(\omega+i e)] \\
& =-\frac{1}{2} \frac{N^{3}-N}{6} \operatorname{Im}[C S(\omega+i e)]=\frac{N^{3}-N}{6} \operatorname{vol}(M), \quad \text { and } \\
\operatorname{Im}\left[S_{0}^{\overline{\mathrm{geom}}}\right] & =-\operatorname{Im}\left[S_{0}^{\mathrm{geom}}\right]=-\frac{N^{3}-N}{6} \operatorname{vol}(M) .
\end{aligned}
$$

In the second line, $T^{a}(a=1,2,3)$ are Pauli matrices and $\rho_{N} \cdot\left(T^{a}\right)$ are generators in the $N$-dimensional irreducible representation. From a simple group theoretical fact

$$
\frac{\operatorname{Tr}\left[\rho_{N} \cdot\left(T^{a}\right) \rho_{N} \cdot\left(T^{b}\right)\right]}{\operatorname{Tr}\left[T^{a} T^{b}\right]}=\frac{N^{3}-N}{6}
$$

the expected $N^{3}$-scaling of $T_{N}[M]$ theory follows. In the third line of (21), we use the fact that the imaginary part of Chern-Simons functional of $\mathcal{A}=\omega+i e$ is equal to the Einstein-Hilbert action with unit negative cosmology constant up to an overall numerical factor [52]. The action for the unique hyperbolic metric is twice of the hyperbolic volume of 3-manifold with a minus sign.

The large $N$ asymptotic behavior of the 1-loop coefficients, $S_{1}^{\text {geom }}$ and $S_{1}^{\overline{\text { geom }}}$, can be analyzed using a following mathematical theorem [53],

$$
\begin{aligned}
& \log \left|\operatorname{Tor}_{R=\rho_{2 m+1}}\left[\mathcal{A}_{N=2}^{\text {geom }}, M\right]\right| \\
& \stackrel{\text { as } m \text { goes to } \infty}{\longrightarrow}-\frac{1}{\pi} m^{2} \operatorname{vol}(M)+o(m) .
\end{aligned}
$$

Here $\rho_{2 m+1}$ is the $(2 m+1)$-dimensional irreducible representation of $\operatorname{sl}(2, \mathbb{C})=s u(2)_{\mathbb{C}}$. Combining the theorem with the following branching rule,

(adjoint of $\operatorname{sl}(N, \mathbb{C}))=\bigoplus_{m=1}^{N-1} \rho_{2 m+1}$ of $\operatorname{sl}(2, \mathbb{C})$,

when the $s l(2, \mathbb{C})$ is embedded into $s l(N, \mathbb{C}) \operatorname{via} \rho_{N}$,

we have following large $N$ behavior of the 1-loop coefficients

$$
\begin{aligned}
\operatorname{Re}\left[S_{1}^{\text {geom }}\right] & =\frac{1}{2} \log \left|\operatorname{Tor}_{R=\text { adjoint }}\left[\mathcal{A}_{N}^{\text {geom }}, M\right]\right| \\
& =\frac{1}{2} \sum_{m=1}^{N-1} \log \left|\operatorname{Tor}_{R=\rho_{2 m+1}}\left[\mathcal{A}_{N=2}^{\text {geom }}, M\right]\right| \\
& =-\frac{1}{2 \pi} \operatorname{vol}(M) \sum_{m=1}^{N-1} m^{2}+o(m)=-\frac{N^{3}+o\left(N^{2}\right)}{6 \pi} \operatorname{vol}(M),
\end{aligned}
$$

and

$$
\operatorname{Re}\left[S_{1}^{\overline{\mathrm{geom}}}\right]=\operatorname{Re}\left[S_{1}^{\mathrm{geom}}\right]=-\frac{N^{3}+o\left(N^{2}\right)}{6 \pi} \operatorname{vol}(M) .
$$

Combining the 3D-3D dictionaries in Table I with the large $N$ analysis in (21) and (25), we finally obtain the following universal large $N$ behavior of the twisted ptns (12)

$$
\begin{aligned}
F_{g, p}^{\mathrm{geom}} & :=-\log \left|Z_{g, p}^{\mathrm{geom}}\left(T_{N}[M]\right)\right| \\
& =\frac{4(1-g) N^{3}+p N^{3}}{12 \pi} \operatorname{vol}(M)+o\left(N^{2}\right), \\
F_{g, p}^{\overline{\mathrm{geom}}} & :=-\log \left|Z_{g, p}^{\overline{\mathrm{geom}}}\left(T_{N}[M]\right)\right| \\
& =\frac{4(1-g) N^{3}-p N^{3}}{12 \pi} \operatorname{vol}(M)+o\left(N^{2}\right) .
\end{aligned}
$$

They nicely match the on-shell actions $I_{g, p}^{\text {Bolt }_{\perp}}$ of Bolt ${ }_{ \pm}$ solution in [10]. The large $N$ computations are summarized in Table II.

\section{CONCLUSION}

In this paper, we probe a large class of $\mathrm{AdS}_{4} / \mathrm{CFT}_{3}$ associated to M5-branes wrapped on 3-manifolds by computing large $N$ twisted ptns. For M2 and D2branes and their Chern-Simons-matter theories, the large $N$ computations have been performed already in [2-11]. 
A nicer feature of our analysis is that we map the large $N$ analysis to a mathematical problem via 3D-3D correspondence which can be solved from known mathematical results, such as (19), (21) and (25). The results hold for any closed hyperbolic 3-manifold $M$ and one does not need to perform the large $N$ analysis for individual $\mathrm{AdS}_{4} / \mathrm{CFT}_{3}$ model associated to each $M$. We hope that the improvement made in our analysis may provide a better way of understanding the subleading corrections to the large $N$ twisted ptns which might be related to quantum corrections to the Bekenstein-Hawking entropy.

\section{ACKNOWLEDGMENTS}

We would like to thank Seok Kim, Sunjin Choi, Chiung Hwang, Jaewon Song, Masahito Yamazaki and Victor Mikhaylov for interesting discussions on related works. This work was initiated while the authors were visiting Asia Pacific Center for Theoretical Physics (APCTP), Pohang for a workshop "Strings, Branes and Gauge theories", 16-25 July 2018. We thank APCTP for hospitality. The work of D. G. was supported by Samsung Science and Technology Foundation under Project No. SSTBA1402-08. The research of N.K. was supported by NRF Grant No. 2015R1D1A1A09059301.
[1] A. Strominger and C. Vafa, Microscopic origin of the Bekenstein-Hawking entropy, Phys. Lett. B 379, 99 (1996).

[2] F. Benini, K. Hristov, and A. Zaffaroni, Black hole microstates in $\mathrm{AdS}_{4}$ from supersymmetric localization, J. High Energy Phys. 05 (2016) 054.

[3] F. Benini, K. Hristov, and A. Zaffaroni, Exact microstate counting for dyonic black holes in $\mathrm{AdS}_{4}$, Phys. Lett. B 771, 462 (2017).

[4] S. M. Hosseini and A. Zaffaroni, Large $N$ matrix models for $3 \mathrm{~d} \mathcal{N}=2$ theories: Twisted index, free energy and black holes, J. High Energy Phys. 08 (2016) 064.

[5] S. M. Hosseini and N. Mekareeya, Large $N$ topologically twisted index: Necklace quivers, dualities, and SasakiEinstein spaces, J. High Energy Phys. 08 (2016) 089.

[6] A. Cabo-Bizet, V. I. Giraldo-Rivera, and L. A. Pando Zayas, Microstate counting of $\mathrm{AdS}_{4}$ hyperbolic black hole entropy via the topologically twisted index, J. High Energy Phys. 08 (2017) 023.

[7] F. Azzurli, N. Bobev, P. M. Crichigno, V. S. Min, and A. Zaffaroni, A universal counting of black hole microstates in $\mathrm{AdS}_{4}$, J. High Energy Phys. 02 (2018) 054.

[8] S. M. Hosseini, K. Hristov, and A. Passias, Holographic microstate counting for $\mathrm{AdS}_{4}$ black holes in massive IIA supergravity, J. High Energy Phys. 10 (2017) 190.

[9] F. Benini, H. Khachatryan, and P. Milan, Black hole entropy in massive type IIA, Classical Quantum Gravity 35, 035004 (2018).

[10] C. Toldo and B. Willett, Partition functions on $3 \mathrm{~d}$ circle bundles and their gravity duals, J. High Energy Phys. 05 (2018) 116.

[11] N. Bobev and P. M. Crichigno, Universal RG flows across dimensions and holography, J. High Energy Phys. 12 (2017) 065.

[12] J. M. Maldacena, The large $N$ limit of superconformal field theories and supergravity, Int. J. Theor. Phys. 38, 1113 (1999); Adv. Theor. Math. Phys. 2, 231 (1998).

[13] S. Gukov and D. Pei, Equivariant Verlinde formula from fivebranes and vortices, Commun. Math. Phys. 355, 1 (2017).

[14] F. Benini and A. Zaffaroni, A topologically twisted index for three-dimensional supersymmetric theories, J. High Energy Phys. 07 (2015) 127.
[15] F. Benini and A. Zaffaroni, Supersymmetric partition functions on Riemann surfaces, Proc. Symp. Pure Math. 96, 13 (2017).

[16] C. Closset and H. Kim, Comments on twisted indices in 3d supersymmetric gauge theories, J. High Energy Phys. 08 (2016) 059.

[17] C. Closset, H. Kim, and B. Willett, Supersymmetric partition functions and the three-dimensional A-twist, J. High Energy Phys. 03 (2017) 074.

[18] M. Pernici, Spontaneous compactification of sevendimensional supergravity theories, Classical Quantum Gravity 2, 673 (1985).

[19] J. P. Gauntlett, N. Kim, and D. Waldram, M five-branes wrapped on supersymmetric cycles, Phys. Rev. D 63, 126001 (2001).

[20] W. P. Thurston, The Geometry and Topology of ThreeManifolds (Princeton University, Princeton, NJ, 1979).

[21] T. Dimofte, S. Gukov, and L. Hollands, Vortex counting and Lagrangian 3-manifolds, Lett. Math. Phys. 98, 225 (2011).

[22] Y. Terashima and M. Yamazaki, SL(2,R) Chern-Simons, Liouville, and gauge theory on duality walls, J. High Energy Phys. 08 (2011) 135.

[23] T. Dimofte, D. Gaiotto, and S. Gukov, Gauge theories labelled by three-manifolds, Commun. Math. Phys. 325, 367 (2014).

[24] J. Yagi, 3d TQFT from 6d SCFT, J. High Energy Phys. 08 (2013) 017.

[25] S. Lee and M. Yamazaki, 3d Chern-Simons theory from M5-branes, J. High Energy Phys. 12 (2013) 035.

[26] C. Cordova and D. L. Jafferis, Complex Chern-Simons from M5-branes on the squashed three-sphere, J. High Energy Phys. 11 (2017) 119.

[27] C. Beem, T. Dimofte, and S. Pasquetti, Holomorphic blocks in three dimensions, J. High Energy Phys. 12 (2014) 177.

[28] T. Dimofte, Complex Chern-Simons theory at level k via the 3d-3d correspondence, Commun. Math. Phys. 339, 619 (2015).

[29] S. Gukov, P. Putrov, and C. Vafa, Fivebranes and 3-manifold homology, J. High Energy Phys. 07 (2017) 071.

[30] S. Gukov, D. Pei, P. Putrov, and C. Vafa, BPS spectra and 3-manifold invariants, arXiv:1701.06567. 
[31] V. Mikhaylov, Teichmuller TQFT vs Chern-Simons theory, J. High Energy Phys. 04 (2018) 085.

[32] D. Gang and K. Yonekura, Symmetry enhancement and closing of knots in 3d/3d correspondence, arXiv:1803.04009.

[33] T. Dimofte, M. Gabella, and A. B. Goncharov, K-decompositions and 3d gauge theories, J. High Energy Phys. 11 (2016) 151.

[34] D. Gang, Y. Tachikawa, and K. Yonekura, Smallest 3d hyperbolic manifolds via simple $3 \mathrm{~d}$ theories, Phys. Rev. D 96, 061701 (2017).

[35] A. Donos, J. P. Gauntlett, N. Kim, and O. Varela, Wrapped M5-branes, consistent truncations and AdS/CMT, J. High Energy Phys. 12 (2010) 003.

[36] D. Gang, N. Kim, and S. Lee, Holography of 3d-3d correspondence at large N, J. High Energy Phys. 04 (2015) 091.

[37] G. D. Mostow, Quasi-conformal mappings inn-space and the rigidity of hyperbolic space forms, Publ. Math. l'IHÉS 34, 53 (1968).

[38] D. Martelli, A. Passias, and J. Sparks, The supersymmetric NUTs and bolts of holography, Nucl. Phys. B876, 810 (2013).

[39] N. A. Nekrasov and S. L. Shatashvili, Bethe/gauge correspondence on curved spaces, J. High Energy Phys. 01 (2015) 100.

[40] H.-C. Kim and S. Kim, Supersymmetric vacua of massdeformed M2-brane theory, Nucl. Phys. B839, 96 (2010).

[41] K. Intriligator and N. Seiberg, Aspects of $3 \mathrm{~d} N=2$ ChernSimons-matter theories, J. High Energy Phys. 07 (2013) 079.
[42] A. Kapustin, B. Willett, and I. Yaakov, Exact results for Wilson loops in superconformal Chern-Simons theories with matter, J. High Energy Phys. 03 (2010) 089.

[43] S. Gukov, Three-dimensional quantum gravity, ChernSimons theory, and the A polynomial, Commun. Math. Phys. 255, 577 (2005).

[44] T. Dimofte, S. Gukov, J. Lenells, and D. Zagier, Exact results for perturbative Chern-Simons theory with complex gauge group, Commun. Num. Theor. Phys. 3, 363 (2009).

[45] S. Gukov and H. Murakami, SL(2,C) Chern-Simons theory and the asymptotic behavior of the colored Jones polynomial, Lett. Math. Phys. 86, 79 (2008).

[46] D. Gang, M. Romo, and M. Yamazaki, All-order volume conjecture for closed 3-manifolds from complex Chern-Simons theory, Commun. Math. Phys. 359, 915 (2018).

[47] D. B. Ray and I. M. Singer, R-torsion and the Laplacian on Riemannian manifolds, Adv. Math. 7, 145 (1971).

[48] S. Gukov and P. Sulkowski, A-polynomial, B-model, and quantization, J. High Energy Phys. 02 (2012) 070.

[49] E. Witten, Quantum field theory and the jones polynomial, Commun. Math. Phys. 121, 351 (1989).

[50] C. Closset, H. Kim, and B. Willett, Seifert fibering operators in $3 \mathrm{~d} \mathcal{N}=2$ theories, J. High Energy Phys. 11 (2018) 004.

[51] D. Gang, V. Mikhaylov, and M. Yamazaki (to be published).

[52] E. Witten, $2+1$ dimensional gravity as an exactly soluble system, Nucl. Phys. B311, 46 (1988).

[53] W. Müller, The asymptotics of the ray-singer analytic torsion of hyperbolic 3-manifolds, in Metric and Differential Geometry (Springer, New York, 2012), pp. 317-352. 